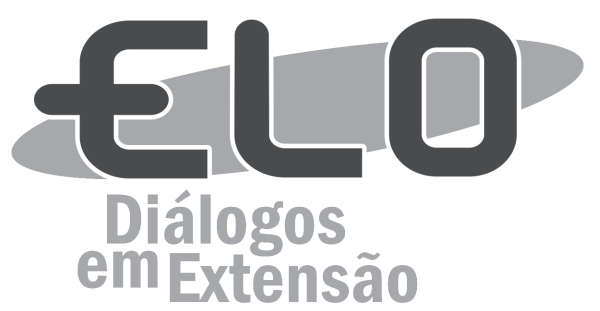

\title{
Método Treino \&Visita: uma alternativa para transferência de tecnologias para cafeicultores das Matas de Minas e promoção do desenvolvimento agricola
}

\author{
Sérgio Maurício Lopes Donzeles ${ }^{1}$, Cláudia Lúcia de Oliveira², Cleide Maria Ferreira Pinto Pinto ${ }^{3}$, Marcelo de \\ Freitas Ribeiro ${ }^{4}$, Sammy Fernandes Soares ${ }^{5}$, Gabriel Braga Violento ${ }^{6}$, Iuri de Freitas Lopes Donzeles ${ }^{6}$
}

\begin{abstract}
Resumo: Objetivou-se transferir tecnologias para cafeicultores das Matas de Minas com fins de adequar as suas propriedades às condições exigidas para certificação pelo programa estadual de certificação "Certifica Minas Café". Utilizou-se o método Treino \& Visita (TEV V) na transferência de tecnologias de construção de fossas sépticas para tratamento de esgoto e de caixas de contenção de águas pluviais. Foram realizados treinamentos para extensionistas sobre saneamento básico e conservação de solos. Sob a supervisão de pesquisadores, os extensionistas treinados fizeram exposição de técnicas sobre construção de fossas sépticas e caixas de contenção aos produtores em unidades demonstrativas, instaladas em propriedades certificadas. Por meio do método TEV é possível capacitar grupos de extensionistas para transferir tecnologias de construção de fossas sépticas e de caixas de contenção além de fortalecer a interação entre pesquisadores e extensionistas.
\end{abstract}

Palavras-chave: Café. Caixa de contenção. Certificação.

Área Temática: Teorias e Metodologias em extensão, Meio Ambiente.

\section{Method Training \& Visit: an alternative to technology transfer to farmers from the Forests of Mines and promoting agricultural development}

Abstract: The objective was to transfer technology to farmers of coffle for purposes of adjusting their properties to the requirements conditions by the certification by the certification. The method used in the transfer of technologies of septic tank construction for wastewater treatment and storm water containment boxes was Training $\mathcal{E}$ Visit (TEV).. Trainings were realized to extension workers about sanitation and soil conservation. Under the supervision of researchers, the trained extension workers did exposure techniques about construction of septic tanks and containment boxes to producers in demonstration units, installed in certified properties. Through the TEV method it is possible to enable extension groups to transfer construction technologies of septic tanks and containment boxes. In addition, strengthen the interaction between researchers and extension workers.

Keywords: Coffee. Containment box. Certification.

Tematic areas: Theories and Methodologies in length, Environment.

\footnotetext{
${ }^{1}$ Eng ${ }^{\circ}$ Agrícola, D.S., Pesq. EPAMIG Zona da Mata, Caixa postal 216 CEP 36570-000 Viçosa MG, Correio eletrônico: slopes@ufv.br

${ }^{2}$ Farmacêutica-Bioquímica, D.S., Pesq. EPAMIG Zona da Mata/Bolsista Fapemig, Caixa Postal 216, CEP 36570-000 Viçosa-MG. Correio eletrônico: clucia@epamig.ufv.br

${ }^{3}$ Enga Agra $^{\text {a }}$ D.S., Pesq. EMBRAPA/EPAMIG Zona da Mata, Caixa postal 216 CEP 36570-000 Viçosa MG. Correio eletrônico: cleide.pinto@epamig.ufv.br

${ }^{4}$ Eng $^{\text {o }}$ Agrônomo, D.S., Pesq. EPAMIG Zona da Mata, Caixa postal 216 CEP 36570-000 Viçosa MG. Correio eletrônico: mribeiro@epamig.ufv.br

${ }^{5}$ Eng $^{\mathrm{O}}$ Agr ${ }^{\circ}$, D.S., Pesq. EMBRAPA/EPAMIG Zona da Mata, Caixa postal 216 CEP 36570-000 Viçosa MG, Correio eletrônico: Correio eletrônico: sammy@epamig.ufv.br

${ }^{6}$ Graduandos Agronomia UFV, Estagiários EPAMIG/UREZM, Caixa postal 216 CEP 36570-000 Viçosa MG.
} 


\section{Método de Entrenamiento y Visita: una alternativa a la transferencia de tecnología a los agricultores de los bosques de Minas y promover el desarrollo agricola}

Resumen: El objetivo fue transferir tecnologias para cafeicultores com el fin de adequar sus propiedades a condiciones exigidas para la certificación "Certifica Minas Café ". En capacitación se utilizo el método de Transferencia y Visita (TEV) de tecnologias de construcción de tanque séptico para tratamiento de aguas residuales y cajas de agua de aguas pluviales.. Fueran realizadas capacitaciones para extensionistassobre el saneamiento y conservación del suelo. Bajo la supervisión de investigadores, los extensionistas capacitados hicieron exposición de técnicas sobre construcción de tanques sépticos y cajas de contención a los productores en unidades de demostración instaladas en propiedades certificadas. A través del método de TEV fue posiblecapacitar grupos de extensionistas para transferir tecnologías de construcción de tanques sépticas y cajas de contención, ademas de fortalecer la interacción entre los investigadores y extensionistas.

Palabras clave: Café. Caja de contención. La certificación.

Áreas Temáticas: Teorías y Metodologías de longitud. Medio Ambiente.

\section{Introdução}

No Brasil, a cafeicultura é uma atividade de grande importância para o cenário internacional e contribui, consideravelmente, para a geração de emprego, receitas cambiais e formação de divisas. Dentre as commodities mais negociadas do mercado, a produção de café é a que apresenta a maior volatilidade. Os estados de Minas Gerais, Espírito Santo, São Paulo e Paraná são os maiores produtores de café sendo o Espírito Santo o maior produtor de café conillon e, Minas Gerais, de café arábica. Em Minas Gerais, estado que abrange um território de $586.528 \mathrm{~km}^{2}$ de área, a cafeicultura apresentou crescimento, principalmente após da década de 1970, quando intempéries climáticas prejudicaram as lavouras paulista e paranaense. Atualmente, o café é o principal produto da agropecuária mineira, com grande importância no cenário econômico, político e social do Estado. A cafeicultura proporciona a geração de empregos diretos e indiretos; desse modo, estimula a permanência do homem no campo e, em consequência, evita-se o êxodo rural.

A cadeia produtiva do café tem a participação de diversas organizações, instituições e centros de pesquisa. Essa integração contribui para o desenvolvimento, o controle e o aperfeiçoamento da atividade cafeeira com reflexo no aumento da área total plantada de café das espécies arábica e robusta no Brasil que atualmente é de 2.267.577,8 hectares. Do total, 341.504,4 hectares (15,06\%) estão em fase de formação e $1.926 .073,4$ hectares $(84,94 \%)$ em fase de produção. Em Minas Gerais está concentrada a maior área plantada com 1.245 .710 mil hectares, com predomínio da espécie arábica $(98,89 \%)$. A área total plantada de café no estado de Minas Gerais representa 54,2\% da área cultivada com café no País. As maiores regiões produtoras do estado são, respectivamente, o Sul e Oeste de Minas Gerais, seguidos de Alto Paranaíba e Triângulo Mineiro, Zona da Mata e Jequitinhonha (OLIVEIRA et al., 2008; IBGE, 2014; CONAB, 2014).

Na região das Matas de Minas, a produção de café é uma das principais atividades agrícolas, nos aspectos de geração de renda e emprego. Nessa região, a cafeicultura é explorada em propriedades predominantemente familiares, em terrenos acidentados, com restrições à mecanização e em solos pobres e desgastados. Dessa forma, para manter a competitividade do café produzido é preciso melhorar a qualidade do produto e ajustar o processo produtivo às exigências do mercado, com a finalidade de agregar valor ao produto.

\section{Programa estadual de certificação de café "certifica minas café"}

Uma opção para os produtores é a sua adesão ao Programa Estadual de Certificação de Café da Secretaria de Estado da Agricultura, Pecuária e Abastecimento, denominado "Certifica Minas Café" (EMATER, 2014). Trata-se de um Programa Estruturador do Governo do Estado de Minas Gerais que visa identificar as propriedades produtoras de café, inclusive orgânico, com objetivos de rastreabilidade, manutenção e melhoria da qualidade como instrumento de valorização dos cafés mineiros; manutenção e conquista de novos mercados por meio da qualidade; manutenção, ampliação dos empregos gerados e aprimoramento do atendimento; manutenção e recuperação das áreas de preservação permanente (APP), reservas legais e manejo adequado de solo; certificação das 
propriedades de café; garantia da melhoria e da gestão dessas propriedades e a inserção dos cafés mineiros nos diferentes mercados de forma a atender a crescente demanda de acordo com os padrões de qualidade (EMATER, 2014).

Para adesão dos produtores à certificação de propriedades cafeeiras, segundo os princípios de boas práticas agrícolas (BPA), há necessidade do cumprimento de normas e critérios. As normas (IMA, 2014b) para o manejo da lavoura abrangem grandes áreas temáticas:

- Material de propagação: deve possuir, obrigatoriamente, certificado de sanidade ou Certificado Fitossanitário de Origem - CFO;

- Áreas de cultivo: devem estar identificadas no local e deverá conter pelo menos um ponto georreferenciado da propriedade, deve existir um mapa ou croqui que delimite a ocupação do solo;

- Controle de pragas e doenças: o uso de agrotóxico, em caso de necessidade, deve ser receitado por um agrônomo. O receituário possibilita o produtor constatar que os agrotóxicos utilizados são registrados para a cultura no MAPA e cadastrado no IMA;

- Irrigação: é obrigatória a outorga de água ou protocolo dentro do prazo de validade;

- Colheita e pós-colheita: resíduos provenientes do processamento via úmida do café devem ter destino adequado. Devem ser adotadas boas práticas de secagem do café.

- Rastreamento: deve existir registro atualizado de serviços, compras, colheita, preparo e secagem, armazenamento e beneficiamento, comercialização de café e outros registros necessários.

- Responsabilidade ambiental: prevê praticas de conservação do solo da propriedade, mediante adoção de técnicas como manejo correto do mato e de caixas de contenção, reutilização da água do processamento via úmida e construção de fossas sépticas na propriedade.

- Conservação das águas: é obrigatório que as fontes de água estejam identificadas em mapas ou croqui da propriedade. A partir do ingresso do produtor no Certifica Minas Café são proibidas intervenções, como barragens ou desvios de cursos d'água. Agroquímicos (agrotóxicos e fertilizantes) não podem ser manuseados em locais que ofereçam risco de contaminação das fontes de água.

- Responsabilidade social: é proibido trabalho forçado e infantil. Deve existir liberdade de organização dos trabalhadores e todo trabalhador deve ter acesso a um sistema de saúde.

- Capacitação: treinamento em segurança no trabalho ou curso com grade similar. Todos os colaboradores devem ter treinamento para realização da atividade.

- Gestão da propriedade: os produtores devem ser orientados e acompanhados durante o processo de certificação.

\section{Certificação de Sistemas de Produção de Café na Região das Matas de Minas}

As instituições de pesquisa situadas ou que atuam na região das Matas de Minas dispõem de tecnologias para melhoria da qualidade do café, dentro dos padrões exigidos pelo mercado. Entretanto, pelo fato de muitas dessas tecnologias não serem utilizadas, os cafeicultores da região não obtêm certificado para seus sistemas de produção. Um dos maiores obstáculos para a adoção de tecnologias pelos agricultores familiares é a dificuldade de entendimento e acesso, o que gera um entrave na transferência de tecnologia (MODESTO JÚNIOR; ALVES, 2009).

O interesse pela certificação é crescente, por possibilitar um canal direto de comercialização entre o produtor e a indústria, com a oferta de um prêmio pela qualidade do produto, que varia de 10 a $25 \%$ sobre o preço do dia. Em 2009, existiam 1.024 propriedades cafeeiras certificadas pelo Programa Estadual de Certificação de Café. Atualmente, existem 1.668 propriedades cafeeiras certificadas, ou seja, houve uma adesão superior a 50\% (IMA, 2014a).

A construção de fossas sépticas e de caixas de contenção de águas pluviais está entre os requisitos exigidos para se obter a certificação (EMATER, 2014), mas são frequentemente negligenciados. Poucas propriedades já certificadas na região de Viçosa possuem fossa séptica e menos da metade dispõe de caixas de contenção. Essa situação pode ser associada ao fato de se tratar de um requisito recomendável e não obrigatório no processo de certificação (IMA, 2014a). Entretanto, a instalação das fossas sépticas para tratamento de esgoto domiciliar são fundamentais para a prevenção de doenças como verminoses 
e endemias (cólera) uma vez que servem de reservatórios para dejetos humanos que seriam lançados diretamente em rios, lagos, nascente ou mesmo na superfície do solo. Já as caixas de contenção de águas pluviais têm como finalidade proteger o solo contra erosões e contra a própria retenção da umidade.

\section{Fossas Sépticas e Práticas de Conservação de Solos}

Dentre os modelos de fossa séptica, aquele construído em alvenaria foi projetado pela Fundação Nacional de Saúde (BRASIL, 2006), e aquele denominado fossa séptica biodigestora, constituído por caixas pré-fabricadas, foi desenvolvido por Novaes et al.(2002). Com a utilização de ambos os modelos, é possível fazer o tratamento de esgoto domiciliar sem contaminar pessoas, animais e corpos hídricos. A fossa projetada pela Funasa (BRASIL, 2006) é indicada para receber o esgoto de pias, banheiro e vaso sanitário, enquanto o modelo desenvolvido pela Embrapa é indicado apenas para receber o esgoto de vaso sanitário (FUNDAÇÃO BANCO DO BRASIL, 2010).

Diante do relevo acidentado da região das Matas de Minas é necessária atenção especial no que diz respeito à utilização de práticas de conservação de solos, com fim de evitar erosão. A maioria dos cafeicultores certificados adota alguma prática de conservação em suas lavouras, notadamente, a manutenção da cobertura vegetal natural, que é periodicamente roçada. Entretanto, em razão de, na região de Viçosa, as estradas de acesso às lavouras serem muito expostas à erosão, a construção de caixas de contenção ou caixas secas ajuda a manter essas estradas em condições transitáveis durante períodos mais longos. As caixas secas visam diminuir a velocidade das águas e seu dimensionamento é feito em relação à largura e inclinação da estrada (LANI et al., 2007).

\section{Metodologia Treino \& Visita}

Várias tecnologias já desenvolvidas pela pesquisa ainda são desconhecidas ou pouco conhecidas pelos produtores, sendo uma das razões a dificuldade de comunicação entre os atores responsáveis pelos processos de geração e de transferência da tecnologia. O método Treino \& Visita (T\&V) caracteriza-se pela atuação conjunta e sistematizada das instituições envolvidas no processo de geração e de transferência de tecnologias, as quais são testadas e validadas nas condições da propriedade em questão, com a participação e o julgamento do produtor (MODESTO JÚNIOR; ALVES, 2009). O método vem sendo aplicado com sucesso nos Estados do Sul do Brasil e a proposta é também de fazer a transferência de tecnologias para cafeicultores em processo de certificação na região das Matas de Minas. Em decorrência de seu sucesso e resultados positivos, é imprescindível a divulgação do método $T \& V$ e sua adoção por instituições de pesquisa e extensão rural. Trata-se de uma ferramenta eficiente e inovadora no processo de Transferência de Tecnologia (TT) e estabelecimento de Redes de TT. Além disso, proporciona um fórum permanente de reciclagem de conhecimentos para os profissionais da área de assistência técnica e extensão rural e permite a retroalimentação do processo de pesquisa (P\&D), que é um dos grandes desafios enfrentados pelos profissionais que atuam na TT (DOMIT et al., 2007). Proporciona ainda, o fortalecimento da interação entre a pesquisa, a assistência técnica e os produtores rurais. Na etapa de "Treino" é feita a capacitação dos agentes da extensão sobre a tecnologia e na etapa "Visita" a tecnologia é validada junto aos produtores (DOMIT et al., 2007). O $\mathrm{T} \& \mathrm{~V}$ funciona, basicamente, pela formação e pelo treinamento de técnicos multiplicadores da assistência técnica e extensão rural, os quais, em contato constante com a pesquisa, formam e treinam grupos organizados de técnicos multiplicadores de campo os quais repassam as tecnologias para grupos organizados de produtores rurais. Dessa maneira, ocorre o fortalecimento dos elos entre a pesquisa, a assistência técnica, a extensão rural e os produtores rurais, o que propicia a retroalimentação periódica de informações e a avaliação dos resultados obtidos. A metodologia adotada é pautada no diagnóstico participativo pelo método $\mathrm{T} \& \mathrm{~V}$ onde o " $\mathrm{T}$ " representa o processo de capacitação que deve ser sistêmico, contínuo e prioritariamente com periodicidade definida e o " $\mathrm{V}$ " representa o processo de Transferência de Tecnologia, que também deve ser sistêmico e desenvolvido com todos os agentes envolvidos nos diferentes níveis do trabalho.

Objetivou-se transferir tecnologias pelo método $T \& V$ para cafeicultores da região das Matas de Minas, com fins de adequar suas propriedades às condições exigidas para certificação pelo Programa Estadual de Certificação "Certifica Minas Café". 


\section{Material e métodos}

O trabalho foi coordenado por profissionais da Empresa de Pesquisa Agropecuária de Minas Gerais (EPAMIG), Unidade Regional Epamig Zona da Mata (UREZM) e Empresa Brasileira de Pesquisa Agropecuária (EMPRAPA) com a participação de profissionais da Universidade Federal de Viçosa (UFV), técnicos extensionistas da Empresa de Assistência Técnica e Extensão Rural do Estado de Minas Gerais (EMATER) da Unidade Regional de Viçosa e cafeicultores da região das Matas de Minas.

A transferência de conhecimentos e de tecnologias foi repassada a técnicos extensionistas e produtores por meio da metodologia Treino e Visita $(\mathrm{T} \& \mathrm{~V})$, por se tratar de uma metodologia contínua e sistêmica.

\section{Treino (T)}

Para os treinamentos foram, inicialmente, elaborados materiais didáticos na forma de apostilas sobre os temas: 1) "Projeto, construção e operação de sistemas de tanques sépticos, unidades de tratamento complementar e disposição final dos efluentes líquidos com base na NBR 7229/93 e NBR 13969/97 (ASSOCIAÇÃO BRASILEIRA DE NORMAS TÉCNICAS, 1993; 1997) e 2) "Lavouras cafeeiras de montanha" distribuídas na forma digital (DVD) para os agentes multiplicadores da extensão.

Foram realizados treinamentos sobre saneamento básico e conservação de solos para técnicos extensionistas da Empresa de Assistência e Extensão Rural (EMATER), pertencentes à Unidade Regional de Viçosa-MG e para cafeicultores certificados pelo Programa Certifica Minas Café. Essa fase foi realizada no auditório da EPAMIG, Unidade Regional da Zona da Mata em Viçosa/MG. A finalidade foi embasar a transferência de tecnologias pertinentes aos temas e implantar unidades demonstrativas com fossas sépticas e caixas de contenção de águas pluviais em propriedades rurais.

\section{Visita (V)}

Os técnicos dos escritórios locais da EMATER acompanhados dos respectivos proprietários das lavouras cafeeiras realizaram visitas técnicas às unidades demonstrativas no município de ViçosaMG, a cada dois meses, durante seis meses, para realizar demonstrações técnicas aos cafeicultores e validação das tecnologias no contexto das propriedades rurais.

\section{Registros de observações, coleta de amostras de água, análises microbiológicas}

Nas ocasiões das visitas foram realizados registros de observações feitas pelos cafeicultores sobre o funcionamento das unidades. Foram também realizadas coletas de amostras da água na saída das fossas sépticas, ou seja, na saída da terceira caixa para avaliação da qualidade microbiológica com 30, 60 e 90 dias após o início do seu funcionamento. A eficiência do sistema de biodigestão foi comprovada por incoculação intencional de bactérias do grupo coliformes na primeira caixa com 60 dias, após a coleta da amostra. As amostras foram analisadas no laboratório de análise de água da EPAMIG, onde foram realizadas a contagem de bactérias dos grupos coliformes totais e fecais por meio da técnica de fermentação em tubos múltiplos (DOWNES; ITO, 2001) também denominada técnica do número mais provável (NMP/100 mL) e por meio de kits Colipaper (Tecnobac).

\section{Resultados e discussão}

Os dois treinamentos realizados para técnicos da EMATER sobre os conhecimentos e tecnologias sobre saneamento básico e conservação de solos incluíram os municípios de Paula Cândido, Araponga, São Miguel do Anta, Ervália, Viçosa, Pedra do Anta, Dores do Turvo, Guiricema, Coimbra e Canaã. Assim, foram formados agentes multiplicadores para transferência dessas tecnologias para cafeicultores incluindo proprietários de unidades certificadas ou participantes do programa "Certifica Minas Café". O treinamento inicial a respeito de saneamento básico teve duração de um dia; dele participaram, como instrutores, um professor pesquisador da Universidade Federal de Viçosa, do Departamento de Fitotecnia e um estudante de doutorado do Departamento de Engenharia Agrícola e Ambiental da UFV. As apresentações teóricas foram realizadas no auditório da EPAMIG-Regional da Zona da 
Mata. No segundo dia, foi feita a parte prática no município de Paula Cândido-MG, também com acompanhamento dos mesmos instrutores da parte teórica, na qual foram demonstradas técnicas sobre construção da fossa séptica biodigestora e fossa séptica com sumidouro (Figura 1A).

O treinamento teórico para técnicos da EMATER sobre "Práticas de conservação de solos" teve duração de um dia e foi realizado por pesquisador da EPAMIG, vinculado a Unidade Regional Epamig Zona da Mata em auditório da empresa em Viçosa-MG. Posteriormente, a demonstração prática foi acompanhada por pesquisadores da EPAMIG sobre técnicas de construção de curvas de nível, localização de estradas e construção de caixas de contenção, construção dos diques de contenção e a formação dos travesseiros, dispostos a cada 20 metros, aproximadamente, e carreadores em nível para contenção da enxurrada (Figura 1B).
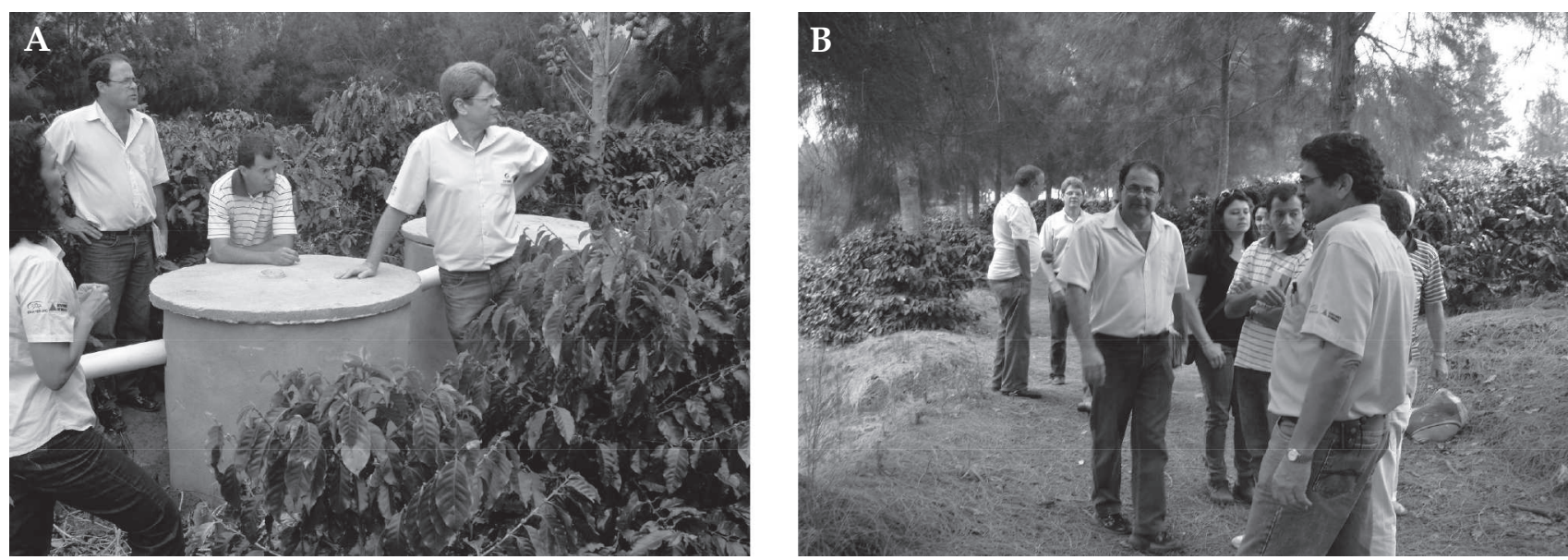

Figura 1 - Demonstrações técnicas de fossa séptica (A) e práticas de conservação de solos (B) para os técnicos da EMATER. Fonte: Arquivo da Epamig de fotografias relativas às atividades do projeto (2014)

Essa primeira etapa, constitui a fase denominada de Treino pelo agente da pesquisa para extensionistas.

Após os treinamentos dos técnicos da EMATER, foram instaladas as unidades demonstrativas em propriedades rurais de cafeicultores certificados, com fossa séptica e com caixas de contenção, nos municípios de Araponga, Ervália, Canaã, Paula Cândido e Oratórios, pelos agentes multiplicadores, ou seja, extensionistas da EMATER local, sob a supervisão dos especialistas da UFV e da EPAMIG. Participaram das unidades demonstrativas os cafeicultores certificados e técnicos de extensão que não participaram dos treinamentos.

Na segunda etapa da metodologia $T \& V$, denominad de visita, o agente de extensão ou multiplicador, que recebeu o treinamento $(\mathrm{T})$, repassou as tecnologias propostas aos produtores rurais por meio da realização de dias de campo, sob a supervisão dos especialistas(Figura 2).

Dessa forma, foi possível formar grupos de extensionistas capacitados para transferir tecnologias de construção de fossas sépticas e de caixas de contenção. Foram construídas 12 fossas e, aproximadamente, 600 caixas de contenção na região das Matas de Minas, área de abrangência proposta no trabalho, o que contribuiu para o aumento expressivo do número de propriedades com fossas sépticas e caixas de contenção na região.

Com relação às amostras de água coletadas na saída do sistema constatou-se contagem de coliformes totais de $1,1 \times 10^{3} / 100 \mathrm{~mL}$ NMP para todas as amostras. A contagem de coliformes fecais foi de $3 \mathrm{x}$ $10^{\circ} / 100 \mathrm{~mL}$ NMP nas amostras coletadas nos dois primeiros meses e $<3 \times 10^{\circ} / 100 \mathrm{~mL}$ NMP nas amostras coletadas nos meses subsequentes o que indicou a eficiência do sistema de biodigestão. A Resolução CONAMA - Conselho Nacional Do Meio Ambiente - $\mathrm{N}^{\circ}$ 20, de 18 de junho de 1986 (CONAMA, 1986), publicada no DOU de 30/7/1986, estabelece que, para águas de classe 2, utilizadas para irrigação de hortaliças e plantas frutíferas, a concentração de coliformes fecais não deve exceder o limite de $10^{3} / 100 \mathrm{~mL}$ de água. Com base nesse padrão, pode-se concluir que o sistema de biodigestão foi eficiente. Portanto, constitui uma alternativa sustentável que contribui para maximização dos recursos disponíveis no âmbito da agricultura familiar sem comprometer a qualidade sanitária para fins de consumo humano além de proporcionar o incremento de produção. 

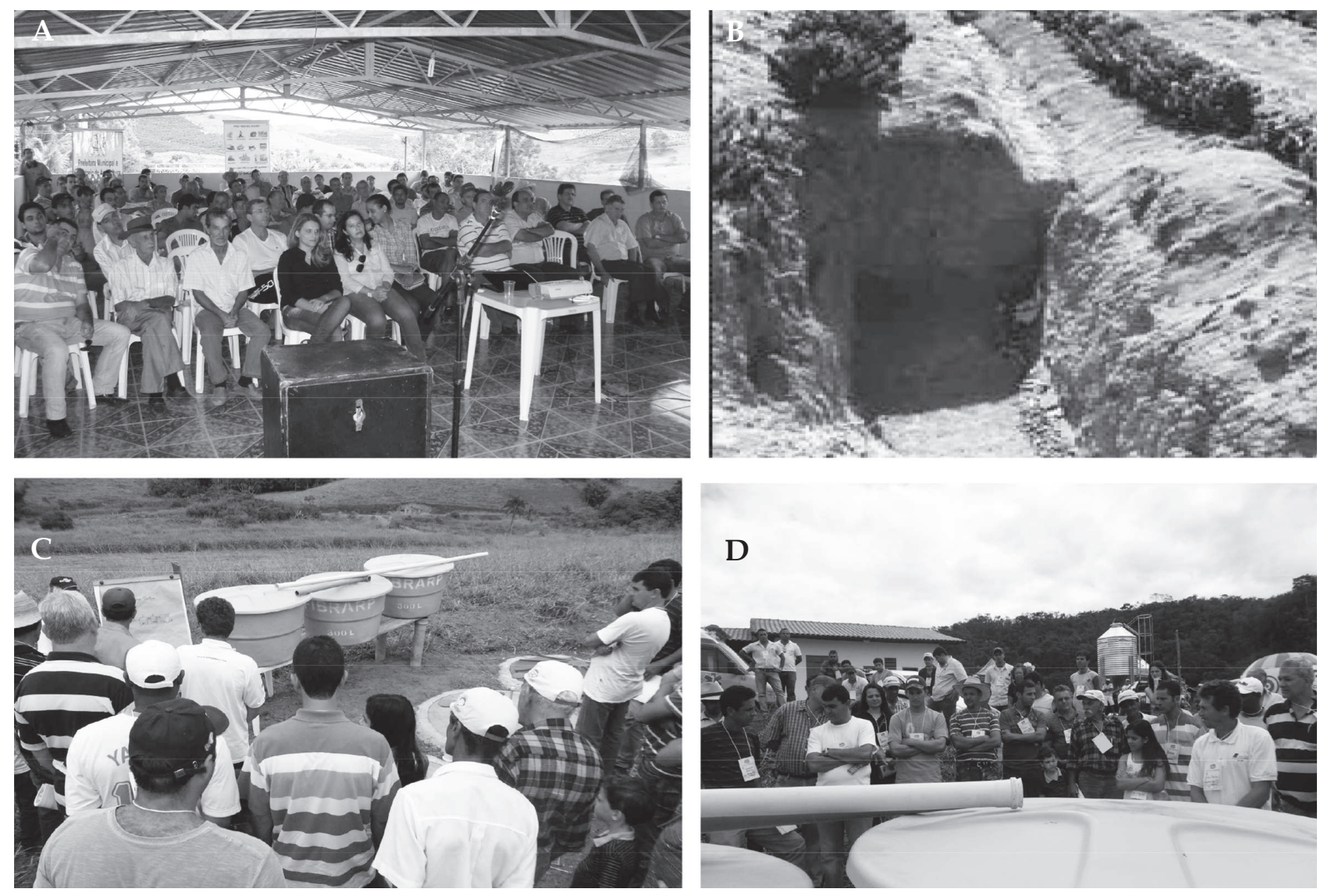

Figura 2 - Dias de Campo realizados pelos extensionistas para produtores incluindo palestra para produtores(A); demonstração de sistema de contenção para preservação do solo (B) e fossas sépticas (C e D)

Fonte: Arquivo da Epamig de fotografias relativas às atividades do projeto (2014)

\section{Considerações finais}

A transferência de tecnologias sobre construção de fossas sépticas e técnicas de conservação do solo por meio do método $T \& V$ na região das Matas de Minas permitiu beneficiar cafeicultores no que se refere à adequação de suas propriedades às condições exigidas para certificação pelo Programa Estadual de Certificação "Certifica Minas Café". Assim, a aplicação desse método de forma bem planejada e conduzida contribui consideravelmente para o desenvolvimento agrícola.

\section{Agradecimentos}

À Fundação de Amparo e Pesquisa do Estado de Minas Gerais - FAPEMIG, pelo apoio financeiro.

\section{Referências bibliográficas}

ASSOCIAÇÃO BRASILEIRA DE NORMAS TÉCNICAS. NBR 7229: Projeto, construção e operação de sistemas de tanques sépticos. 1993. Disponível em: < http://www.deco.ind.br/roman_eng/ NBR_7229.pdf>. Acesso em: 3 dez. 2014.

ASSOCIAÇÃO BRASILEIRA DE NORMAS TÉCNICAS. NBR 7229: Tanques sépticos - Unidades de tratamento complementar e disposição final dos efluentes líquidos - Projeto, construção e operação. 1997. Disponível em: <http://www.acquasana.com.br/legislacao/nbr_13969.pdf>. Acesso em: 3 dez. 2014.

BRASIL. Fundação Nacional de Saúde. Manual de orientações técnicas para elaboração de projeto de melhorias sanitárias domiciliares. $1^{a}$ reimpressão. Brasília: Funasa, 2006. 68 p. Disponível em: <http:/ /www.eadadm.ufsc.br/ ftpcursos/saude/2008/biblioteca/engenhariadasaudepublica/ Mnl\%20Melhorias\%20Sanitarias.pdf>. Acesso em: 26 nov. 2014. 
CONAB - Companhia Nacional de Abastecimento. Acompanhamento da Safra Brasileira de Café, Safra 2014, Terceiro Levantamento, Brasília, set. de 2014 Brasília: v. 1, n. 3, 2014. Disponível em: < http:/ / w w w . c o n a b. g o v. b r / O l a l a C M S / u p l o a d s / a r q u i v o s / 14_09_16_08_47_43_boletim_setembro_2014.pdf>. Acesso em: 26 nov. 2014.

CONAMA - Conselho Nacional do Meio Ambiente. Resolução no 20 de 18 de julho de 1986. RESOLVE estabelecer a seguinte classificação das águas, doces, salobras e salinas do Território Nacional. Diário Oficial [da] República Federativa do Brasil DOU, Brasília-DF, 30 jul. 1986, Seção 1, p.68. Disponível em: < http://www.jusbrasil.com.br/diarios/3549891/pg-68-secao-1-diario-oficial-dauniao-dou-de-30-07-1986>. Acesso em: 26 nov. 2014.

DOMIT, L. A.; LIMA, D. de; ADEGAS, F. S.; DALBOSCO, M.; GOMES, C.; OLIVEIRA, A. B. de; CAMPANINI, S. M. S. Manual de Implantação do Treino e Visita (TEV) / - Londrina: Embrapa Soja, 2007. 86p. - (Documentos / Embrapa Soja, ISSN 1516-781X; n.288) Disponível em < http:// ainfo.cnptia.embrapa.br/digital/bitstream/item/80261/1/Manual-de-implantacao-do-treino-evisita-V-T.pdf>. Acesso em: 26 nov. 2014.

DOWNES, F.P.; ITO, K. Compendium of methods for the microbiological examination of foods. 4.ed. Washington: American Public Health Association - APHA, 2001, 676p.

EMATER - MG. Programa Estadual de Certificação de Café. Manual do Produtor. Belo Horizonte: EMATER, Versão 2. p.9. Disponível em: < http://www.ima.mg.gov.br/material-curso-cfo-cfoc/doc_details/ 1113-manual-do-produtor-cafe-> . Acesso em: 27 nov. 2014.

FUNDAÇÃO BANCO DO BRASIL. Fossa Séptica Biodigestora. 32 p. 2010. Disponível em: < http:// www.fbb.org.br/data / files / BD / A 2/39 / CB / 4205A31009818793 BD983EA 8 / Cartilha\%20Fossas\%20Septicas\%20Biodigestoras.pdf/>. Acesso em: 3 dez. 2014.

IBGE, Instituto Brasileiro de Geografia e Estatística. Banco de Dados Agregados - Sistema de Recuperação Automática - SIDRA. Brasília, 2009. Disponível em: < http://www.sidra.ibge.gov.br/ >. Acesso em: 26 abr. 2014.

IMA. Instituto Mineiro de Agropecuária. Café: Normas da Certificação. Disponível em: < http:// www.ima.mg.gov.br/certificacao/cafe>. Acesso em: 3 dez. 2014a.

IMA. Instituto Mineiro de Agropecuária. Café: Propriedades cafeeiras certificadas.. Disponível em: < http://www.ima.mg.gov.br/portarias/doc_details/700-propriedades-cafeeiras-certificadas>. Acesso em: 26 nov. 2014b.

LANI, J. A.; BRAGANÇA, S. M.; PREZOTTI, L. C.; MARTINS, A. G.; DADALTO, G. G. Preparo, manejo e conservação do solo. In: FERRÃO, R. G.; FONSECA, A. F. A. da; BRAGANÇA, S. M.; FERRÃO, M. A. G.; MUNER, L. H. de. Café conilon. Vitória: Incaper, 2007. p. 279-295. Disponível em < http:// www.sidalc.net/cgi-bin/wxis.exe/?IsisScript=orton. $x$ is\&B1=Buscar\&formato=1\&cantidad =50\&expresion=TRAIT\%20MORPHOLOGIQUE\%20DU\%20SOL>. Acesso em 26 nov. 2014.

MODESTO JÚNIOR, M.S.; ALVES, R.N.B. Treino e Visita como Processo de Transferência de Tecnologia para Produção de Mandioca em Moju, PA. Revista Raízes e Amidos Tropicais, Volume Especial. In: XIII Congresso Brasileiro da Mandioca- Botucatu. p.574-580. 2009. Disponível em: < http://energia.fca.unesp.br/index.php/rat/article/view/1400/719> Acesso em: 27 nov. 2014

NOVAES, A.P.; SIMÕES, M.L.; NETO, L.M.; CRUVINEL, P.E.; SANTANA, A.; NOVONTNY, E.H.; SANTIAGO, G.; NOGUEIRA, A.R.A. Utilização de uma fossa séptica biodigestora para melhoria do saneamento rural e desenvolvimento da agricultura orgânica. São Carlos-SP: Embrapa Instrumentação Agropecuária, 2002. 5p. (Embrapa Instrumentação Agropecuária. Comunicado Técnico 46). Disponível em < http://saneamento.cnpdia.embrapa.br/tecnologias/Comunicado_Tecnico-462002.pdf>. Acesso em: 26 nov. 2014.

OLIVEIRA, A. D. A. S.; GOMES, M. F. M.; RUFINO, J. D. S. L.; JÚNIOR, A. G. D. S.; GOMES, S. T. Estrutura e Dinâmica da cafeicultura em Minas Gerais. Revista de Economia, v. 34, n. 1, p. 119-142, 2008. Disponível em: < http://ojs.c3sl.ufpr.br/ojs2/index.php/economia/article/view/7451/ 8042>. Acesso em: 26 nov. 2014. 